\title{
Fuzzy Logic Based Induction Motor Condition Monitoring
}

\author{
Prof. Dr. Hossam E. A. Talaat \\ Department of Electrical Power \\ and Machines Engineering \\ Ain Shams University \\ Hossam_Talaat@eng.asu.edu.eg
}

\author{
Dr. Mohamed Ezzat \\ Department of Electrical Power \\ and Machines Engineering \\ Ain Shams University \\ moh_ezzat@yahoo.com
}

\author{
Ahmed Mohamed Saleh \\ Department of BU LPLS Engineering \\ ABB Egypt \\ Student Member IEEE \\ Ahmed.M.Saleh@eg.abb.com
}

\begin{abstract}
This paper introduces a new technique for condition monitoring of motor control centres, to define the motor status and reduce the failure rate of AC motors.

Motor stresses defined, and categorized into loading stresses and supply stresses and then two factors are introduced to assess the motor condition, loading stress factor and supply stress factor, using these two factors a reliable motor index is introduced, which defines well the motor status and helps the plant operator to develop an effective predictive and preventative maintenance program.
\end{abstract}

An intelligent fuzzy logic motors condition monitoring system is developed, MATLAB/Simulink model is built to simulate the proposed system.

\section{Index Terms - AC Motor, Condition Monitoring, Fuzzy logic}

\section{INTRODUCTION}

Induction motors are the workhorses of any industrial plant. Typical motor applications include pumps, fans, compressors, mills, shredders, extruders, de-barkers, refiners, cranes, conveyors, chillers, crushers, and blowers. Statistics have shown that despite their reliability and simplicity of construction, annual motor failure rate is conservatively estimated at $3-5 \%$ per year, and in extreme cases, up to $12 \%$, as in the Pulp and Paper industry [1] - [2]. Downtime in a factory can be very expensive and, in some instances, may exceed the cost of motor replacement. Proper machine protection is required to minimize the motor failure rate, prevent damage to associated equipment and to ensure both personnel safety and production targets.

Modern trends in motor protection are moving in the direction of using the microprocessor based relays on the protection of motors which provides a lot of motor fault protection and enhanced monitoring of motor condition.[2] - [5].

The use of microprocessor based relays provides the ability to connect to higher level systems such as distributed control system (DCS) and open process control (OPC) servers, which allows the relay to communicate its operational condition and status to motor control centers (MCC) control rooms, and increase the value of the condition monitoring system [4].

This can be used for real-time monitoring of motors that can give figures for plant operator to detect motor problems before they occur. The maintenance performed on the motors is no longer reactive. Predictive and preventative measures can now be performed to prevent motor failure and damage, allowing plant operators to extend the life of their motors.

That is why MCC management relays manufacturers focus on communicating all motor parameters to the plant control room to give the plant operators all the figures of the motor stresses and help them to make the predictive decision based on their experience. [6]

Service plant operator experts have long had methods for analyzing customer diagnostic information. These methods are very effective in the right expert's hands. So, this collected information is only useful on the hands of a limited number of experts, or for a limited number of customer processes. [7]

Form that, the idea comes to create a factor for implementing these collected experience into a factor that capture and analyze data so effectively that they can be used by less experienced engineers and for multiple customer processes.

Hence, These paper introduces the idea of having a new factor for motor condition monitoring, this factor gives a figure of the status of the motor based on stresses on the motors "Motor Stress factor "

This factor may be a daily stress factor or a yearly stress factor, this depend on if we are using this factor on a daily operation or we are using it on the yearly maintenance, for example for expecting the maintenance periods, or to avoid breaking of the insulation we need yearly stress factor .

\section{PROBLEM FORMULATION}

Motor stresses arise due to a lot of conditions: Overload, over voltage, under voltage, Temperature, and the current unbalance of the motor.

By classifying these conditions into two categories, two assessment modules are driven:

A. Loading stresses: (First assessment module)

This arises due to loading problems including (Overloading, Increasing of number of starts in certain time, and number of trips).

The first assessment module implementation is based on three factors : 


\section{1- Heat rise:}

One of the most important and critical stress on the motor is stresses exerted on the motor winding due to increase of its temperature, The main reason of this is the overloading of the motor, the heat rise factor is induced to give indication of the degree of loading of the motor and its limits are given by thermal limit curve of the motor supplied by its manufacturer. As the current increases for a certain time we can get the stress factor by comparing the time with the maximum time that can the motor withstand at this current according to the thermal limit curve of the motor[2] - [5].

So we will use thermal model of the motor to determine the thermal stress on it, the time at certain current with respect to the max withstand time (Trip time ) will give an indication of how much thermal stress is exerted on motor as per equations (1), (2).

$$
\begin{aligned}
& \text { Stress }=\mathrm{t} / \mathrm{t}_{\max } \\
& \mathrm{t}_{\max }(\mathrm{I})=87.4 . \mathrm{CM} /\left(\mathrm{I}^{2}-1\right) \\
& \mathrm{t}_{\max }(\mathrm{I})=\text { trip time seconds } \\
& \mathrm{CM}=\text { Curve multiplier }
\end{aligned}
$$

2- Number of starts per time:

During each motor start a current value of about 6-8 times the motor nominal current passes through the motor winding, and in case of increasing the number of consecutive starts this consecutive starts have a thermal stresses on the motor winding, taking it into account in assessment module will help in inhibiting the starting in case of increased number of starts which will lead to higher temperature rise and higher stress on the motor.

Defining the maximum number of starts will depend mainly on the manufacturer of the motor.

\section{3- Number of trips:}

It is a very good indication of the stress on motors that arises due to faults actually prevented by the protection system, this helps greatly in the daily and yearly stress factor.

The first assessment module will formulate the loading stress factor as shown in fig.1

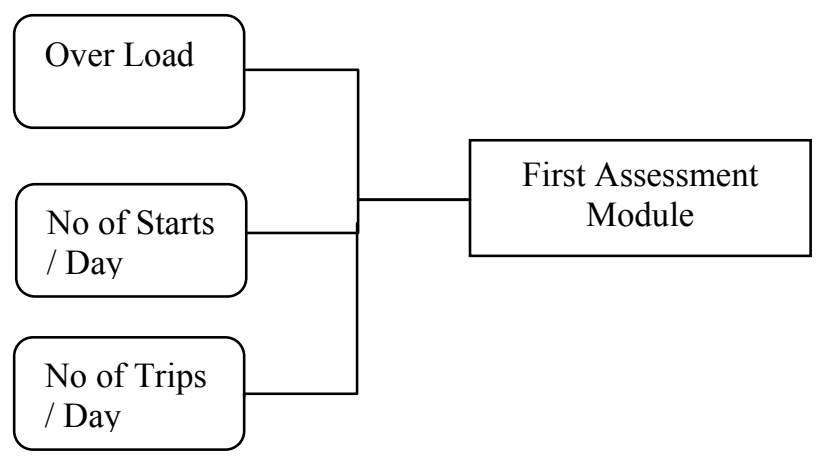

B. Supply stresses: (Second Assessment Module)

This arises due to the stress on motor as a result of supply side problems including (Overvoltage, under Voltage and Voltage Unbalance)

\section{1- Over Voltage:}

High voltage on a motor tends to push the magnetic portion of the motor into saturation. This causes the motor to draw excessive current in an effort to magnetize the iron beyond the point to which it can easily be magnetized. This generally means that the motors will tolerate a certain change in voltage above the design voltage but extremes above the designed voltage will cause the amperage to go up with a corresponding increase in heating and a shortening of motor life.

For example, older motors were rated at 220/440 and had a tolerance band of plus/minus $10 \%$. Thus, the voltage range that they can tolerate on the high voltage connections would be 396 to 484 . Even though this is the so-called tolerance band, the best performance would occur at the rated voltage. The extreme ends, either high or low, would be putting unnecessary stress on the motor. [10]

\section{2- Under Voltage:}

When electric motors are subjected to voltages, below the nameplate rating, some of the characteristics will change slightly and others will change more dramatically. A basic point is, to drive a fixed mechanical load connected to the shaft, a motor must draw a fixed amount of power from the power line. The amount of power the motor draws is roughly related to the voltage times current (amps).

Also, the starting torque, pull-up torque, and pull-out torque of induction motors, all change based on the applied voltage squared. Thus, a $10 \%$ reduction from nameplate voltage (100\% to $90 \%, 230$ volts to 207 volts) would reduce the starting torque, pull-up torque, and pull-out torque by a factor of $.9 \times .9$. The resulting values would be $81 \%$ of the full voltage values. At $80 \%$ voltage, the result would be $.8 \times .8$, or a value of $64 \%$ of the full voltage value. [10]

In this case, it is easy to see why it would be difficult to start "hard-to-start" loads if the voltage happens to be low. Similarly the motor's pull-out torque would be much lower than it would be under normal voltage conditions.

Generally, low voltage can cause high currents and overheating which will subsequently shorten motor life. Low voltage can also reduce the motor's ability to get started and its values of pull-up and pull-out torque. [10] 


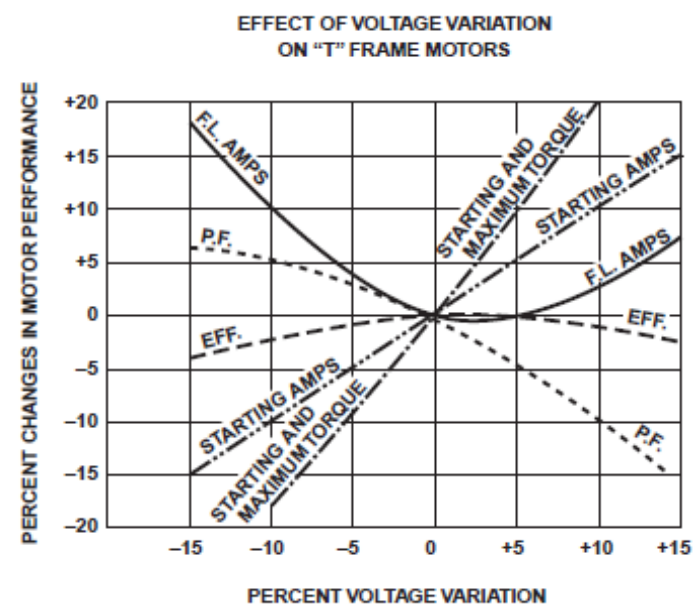

Fig. 2 General effects of high and low voltage

The graph shown in Figure 2 is widely used to illustrate the general effects of high and low voltage on the performance of "T" frame motors. It is okay to use the graph to show "general" effects on motors.

\section{3- Voltage unbalance:}

Unbalancing in the voltage source can cause excessive losses, heating, noise, vibration, torsional pulsations, slip, and motor accelerating torque, detecting of unbalancing in the voltage applied is important, and must be taken into account in our proposed system.

The second assessment module will formulate the supply stress factor as shown in fig. 2

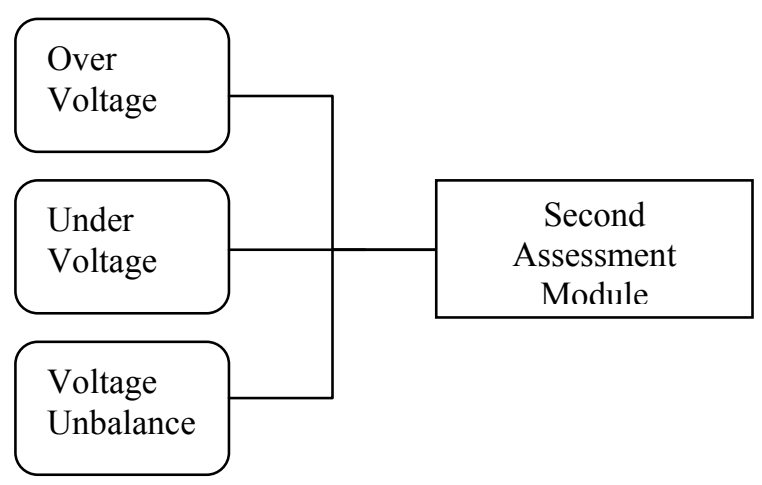

Fig. 3 Second Assessment module

Now to get the total stress factor from the two stress factors we already have due to supply and loading problems, we can also use fuzzy logic as shown figure 4 .

The motor stress factor may have different types according to the application of using of it and the duration of monitoring its condition:
1. Daily Stress Factor :

This will describe the daily status of the motor over 24 hour and can be used in a lot of intraday applications like alternation between spare motors.

\section{Yearly stress factor}

This will be used to assess the motor condition over years of operation and it is used to have a reliable proactive maintenance of motor.

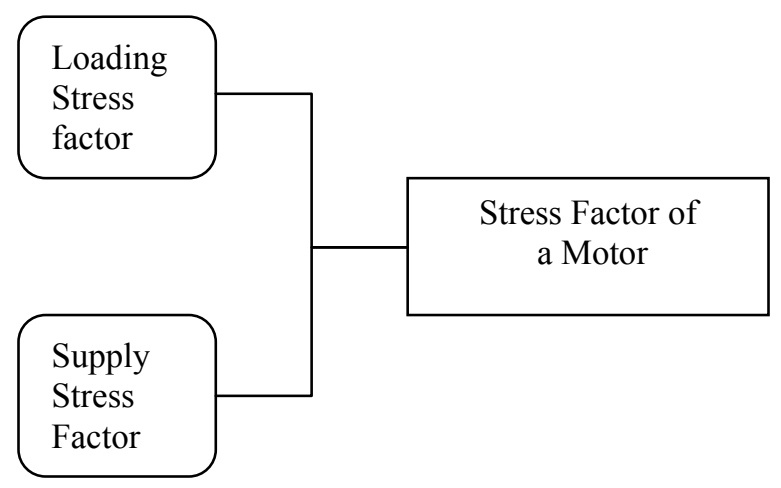

Fig. 4 Stress Factor

\section{SIMULATION MODEL}

These factors are not dependent on each other and there is a lot of conflicts between them, So fuzzy logic is chosen to model this system to get the stress factor parameters for a certain motor.

The problem we have shows a lot of parameters affecting the stress status of the motor and by investigating the different ways that we can use from the intelligent methods, it is found that the best system is the fuzzy logic system .

Fuzzy logic helps our system to make a model for learning through experience that incidental to human being and to representation of vague concepts in mathematical expressions. For that, rule base is created. This rule base may include observation, experience and mathematical equation information in regard to examined issue.

Our aim in the below is to have the rule base built well and comprehensive, to get so precise and accurate results.

The below table is used to describe the accumulation of the human experience to get the value of the loading stress factor.

The first rule base will be created using the three below inputs:

1- The heat rise is formulated by three membership functions; Heat Rise Low (HRL), Heat Rise Medium (HRM) and Heat Rise High (HRH). Fig. 5 shows the membership functions of the heat rise. 


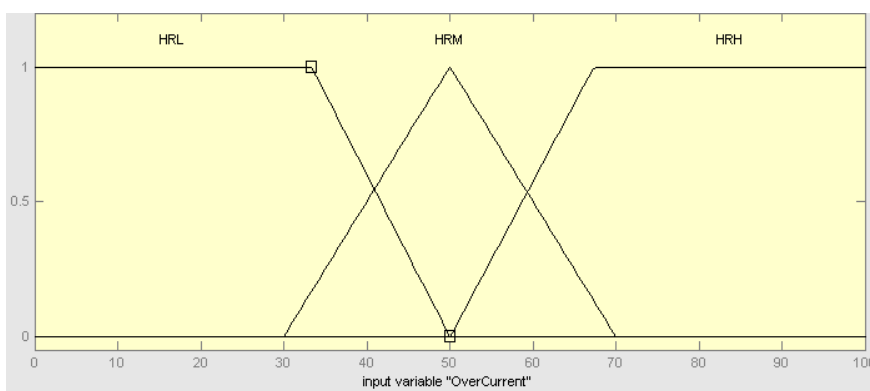

Fig. 5 Heat rise membership function

2- The number of starts per day will be formulated by three membership functions; Number of starts per day Low (NSL), Number of starts per day Medium (NSM) and Number of starts per day High (NSH). Fig. 6 shows the membership functions of number of starts per day.

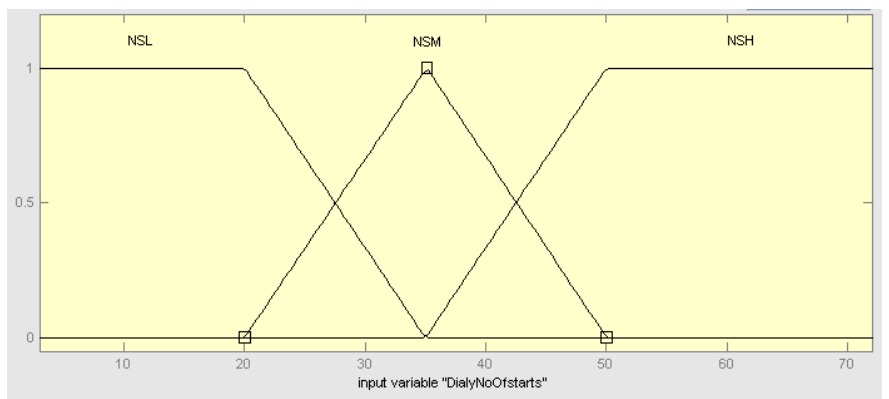

Fig. 6 number of starts per day membership function

3- The number of trips per day will be formulated by three membership functions; Number of trips per day Low (NTL), Number of trips per day Medium (NTM) and Number of starts per day High (NTH). Fig. 7 shows the membership functions of number of trips per day.

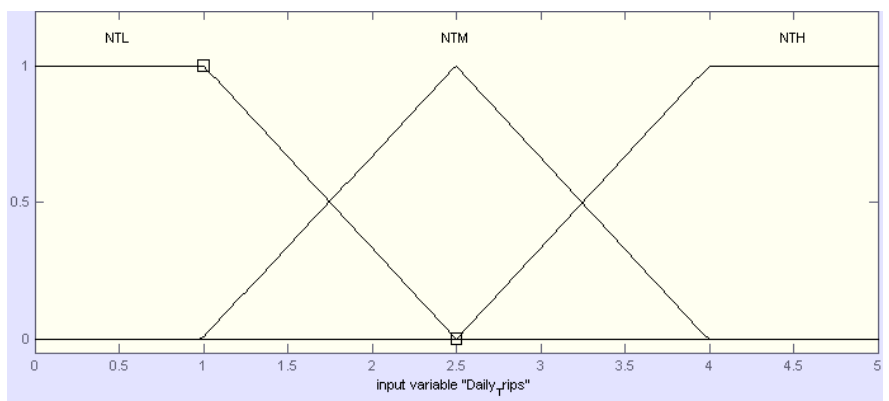

Fig. 7 Number of trips per day membership functions

The complete system for monitoring loading stresses will be as shown in Fig. 8.

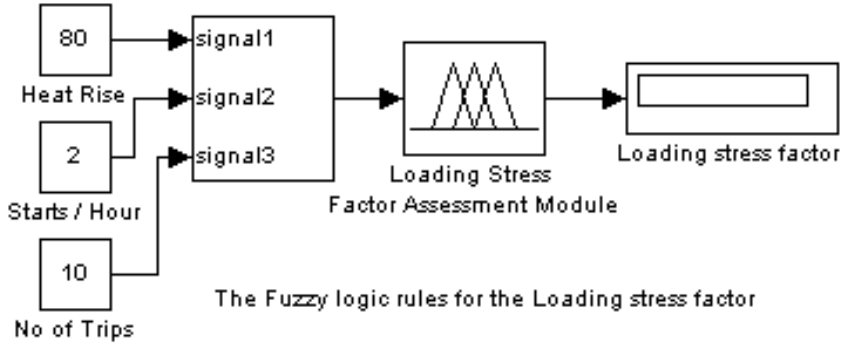

Fig. 8 Loading stress factor model

Then the second rule base will be created using the three below inputs:

1- The over voltage function will be formulated by three membership functions; Over Voltage low (OVL), Over Voltage Medium (OVM) and Over Voltage High $(\mathrm{OVH})$. Fig. 9 shows the membership functions of over voltage.

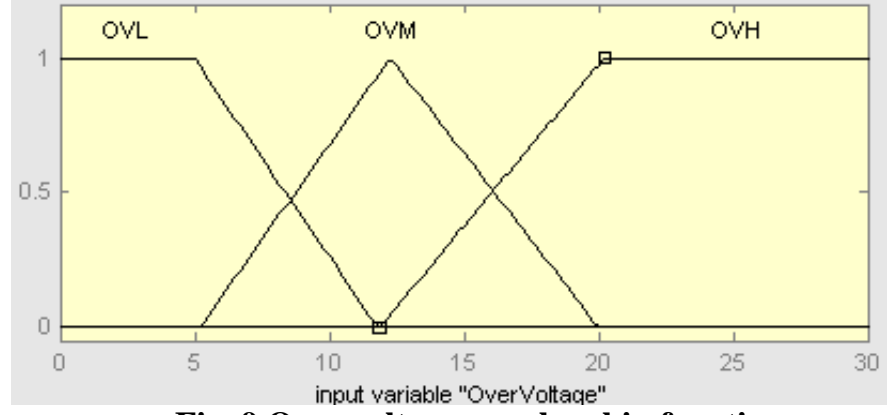

Fig. 9 Over voltage membership function

2- The under voltage function will be formulated by three membership functions; under Voltage low (UVL), Under Voltage Medium (UVM) and Under Voltage High (UVH). Fig. 10 shows the membership functions of under voltage.

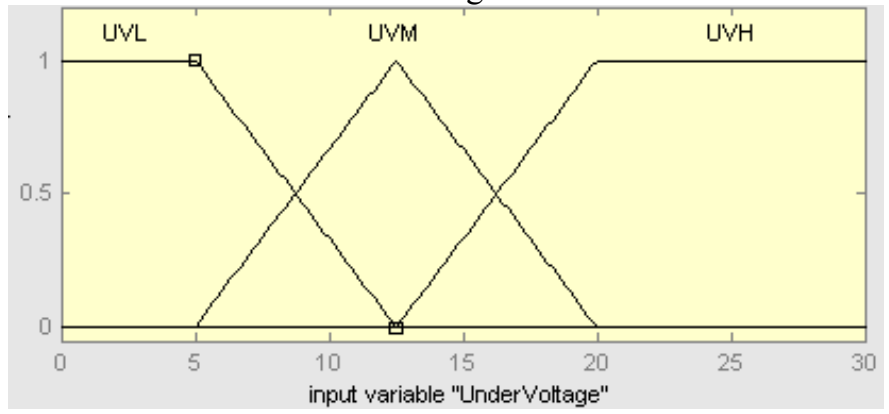

Fig. 10 under voltage membership function

3- The voltage unbalance function will be formulated by three membership functions; voltage unbalance low (UVL), voltage unbalance medium (UVM) and voltage unbalance high (UVH). Fig. 11 shows the membership functions of voltage unbalance. 


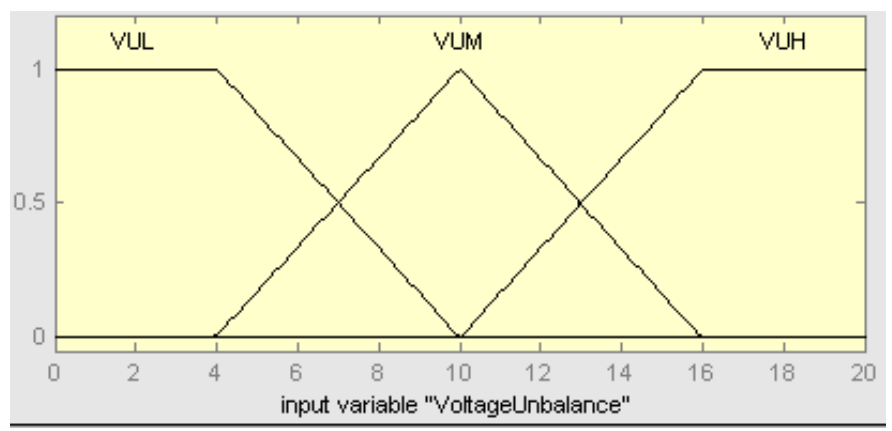

Fig. 11 Voltage Unbalance membership function

The complete system for monitoring supply stresses will be as shown in Fig. 12.

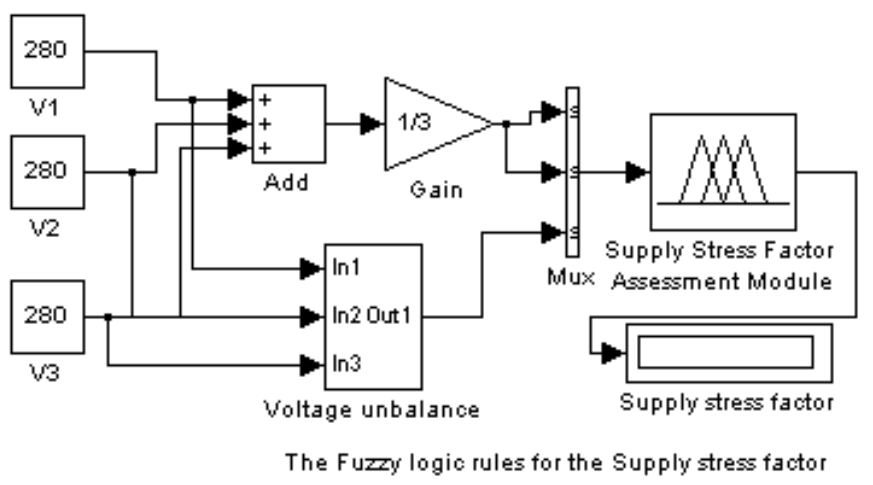

Fig. 12 Supply stress factor model

The third rule base will be created using the two below inputs:

1- Loading stress factor.

2- Supply stress factor.

Then we can build the rules as per table 1 ; where the results of the systems will be Stress Very Low (SVL ), Stress Low (SL ), Stress Medium (SM), Stress High (SH) and Stress Very High (SVH).

TABLE 1

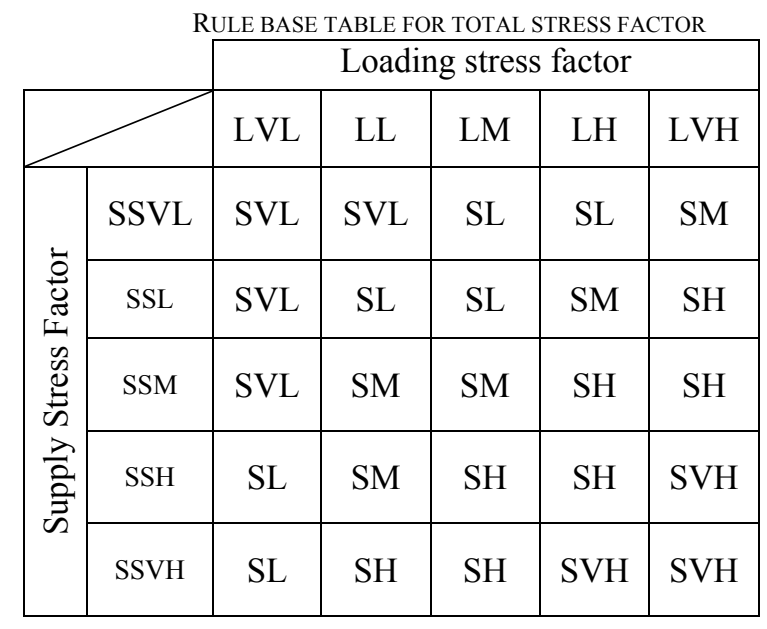

Using MATLAB/SIMULINK

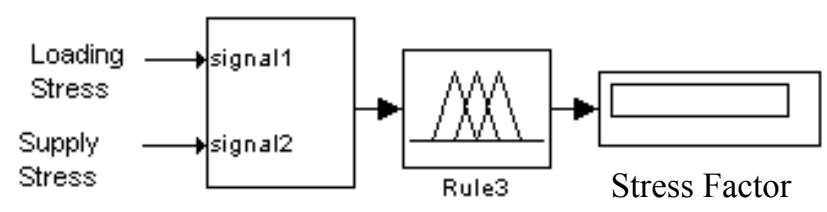

Fig. 13 Total stress factor model

IV. RESULTS

Checking the results of the system:

Now let's test our system on a low voltage motor with the following parameters.

For heat rise range from 0 to $100 \%$, Number of trips per day from 0 to 5 , number of starts per day from 0 to 75 .

Now using SIMULINK we can get the following results for loading stress of the motor

TABLE 2

RESULTS FOR LOADING STRESS FACTOR

\begin{tabular}{|c|c|c|c|c|}
\hline \multicolumn{5}{|c|}{ Rule Base 1} \\
\hline $\begin{array}{l}\text { Rule } \\
\text { No }\end{array}$ & $\begin{array}{c}\text { Heat } \\
\text { Rise (\%) }\end{array}$ & Starts/Hour & $\begin{array}{l}\text { Daily } \\
\text { Trips }\end{array}$ & $\begin{array}{c}\text { Loading } \\
\text { Stress } \\
\text { Factor }(\%)\end{array}$ \\
\hline 1 & 10 & 10 & 1 & 11.36 \\
\hline 2 & 30 & 10 & 1 & 18.94 \\
\hline 3 & 40 & 10 & 1 & 24.89 \\
\hline 4 & 50 & 10 & 1 & 29.67 \\
\hline 5 & 60 & 10 & 1 & 45.76 \\
\hline 6 & 10 & 25 & 1 & 11.36 \\
\hline 7 & 30 & 25 & 1 & 36.7 \\
\hline 8 & 40 & 25 & 1 & 40.27 \\
\hline 9 & 50 & 25 & 1 & 44.98 \\
\hline 10 & 60 & 25 & 1 & 52.22 \\
\hline 11 & 70 & 25 & 1 & 60.07 \\
\hline 12 & 10 & 40 & 1 & 55.05 \\
\hline 13 & 30 & 40 & 1 & 61.58 \\
\hline 14 & 40 & 40 & 1 & 66.12 \\
\hline 15 & 50 & 40 & 1 & 73 \\
\hline 16 & 60 & 40 & 1 & 75.05 \\
\hline 17 & 70 & 40 & 1 & 81.01 \\
\hline 18 & 10 & 60 & 1 & 55.78 \\
\hline 19 & 30 & 60 & 1 & 61.5 \\
\hline 20 & 40 & 60 & 1 & 66.12 \\
\hline 21 & 50 & 60 & 1 & 72.86 \\
\hline 22 & 60 & 60 & 1 & 75.05 \\
\hline 23 & 70 & 60 & 1 & 81.26 \\
\hline 24 & 10 & 60 & 5 & 69.77 \\
\hline 25 & 30 & 60 & 5 & 74.54 \\
\hline
\end{tabular}




\begin{tabular}{|l|r|r|r|r|}
\hline 26 & 40 & 60 & 5 & 80.37 \\
\hline 27 & 50 & 60 & 5 & 88.43 \\
\hline 28 & 60 & 60 & 5 & 88.7 \\
\hline 29 & 70 & 60 & 5 & 88.9 \\
\hline
\end{tabular}

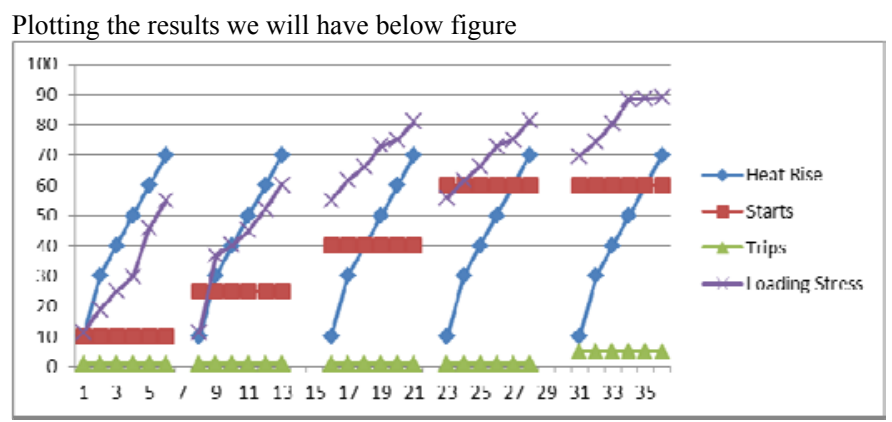

We can notice that:

1. Increase the heat rise increases the loading stress.

2. Increase the number of starts per day increases the loading stress significantly.

3. Increase the number of trips have an effect of increase the loading stress .

\section{CONCLUSION}

In this paper, a fuzzy logic based condition monitoring system that detects the status of the induction motor is developed. A Simulink model is implemented for getting the induction motor status in over voltage, over current, temperature, voltage unbalance, current unbalance and low voltage situations. Stress factor of ac induction motor was introduced as a factor for the status of the motor which can be used by less experienced engineers and for multiple customer processes.

\section{REFERENCES}

[1] M.L Sin , W.L Soong and N. Ertugrul, "Induction machine on-line condition monitoring and fault diagnosis,"

[2] W.Premerlani et al, "Fundamental of motor thermal model and its application in motor protection", 58th Annual Conference for Protective Relay Engineers, pp 127-142, July 2005.

[3] G.Baurand , V. Moliton, "The protection of LV motors," Cahier Technique no. 211 , Schneider Electric.

[4] IEEE Guide for AC motor protection., March 2000.

[5] E. Gaucheron, "Electric motors ... and how to improve their control and protection," Cahier Technique no. 207, Schneider Electric.

[6] Philippe D'Hooghe, John T.Phillipson, "Use and evaluation of smart MCC equipment," IEEE, 1998.

[7] Kevin Starr, "Expert access anytime, anywhere," ABB Review 2/2012 Trends in technology.

[8] Liu Hua , Ding Weiguang ,"The Performance Improvement of Condition Monitoring for Induction Motor based on Neural Network," Proceedings of the IEEE International Conference on Mechatronics and Automation ,2009, Changchun, China.

[9] Davar Mirabbasi, Ghodratollah Seifossadat, Mehrdad Heidari, "Effect of Unbalanced Voltage on Operation of Induction Motors and Its Detection," Electrical and Electronics Engineering conference, IEEE, 2009

[10] Edward Cowern, “ Motors and Drives ," BALDOR

[11] IEEE Guide for the Presentation of Thermal Limit Curves for Squirrel Cage Induction Machines, IEEE Std 620 (R2008)

[12] Jose Policarpo G. de Abreu and Alexander Eigeles Emanuel, "Induction Motor Thermal Aging Caused by Voltage Distortion and Imbalance: Loss of Useful Life and Its Estimated Cost," IEEE transactions on industry applications, vol. 38,no.1, Janyary2002.

[13] Pragasen Pillayand Marubini Manyage, "Loss of Life in Induction Machines Operating With Unbalanced Supplies," IEEE transactions on energy conversion, Vol.21, No.4, December 2006.

[14] Curtis Lanham, "Understanding the Tests that are Recommended for Electric Motor Predictive Maintenance," Baker Instrument.

[15] O.Uyar, M. Cunkas, "Design of fuzzy logic based motor protection system," 6th International Advanced Technologies Symposium (IATS'11), May 2011, Elazığ, Turkey.

[16] K.V. Vamsi Krishna, Effects of unbalance voltage on induction motor current and its operation performance, Lecon Systems.

Arijit Banerjee, Arvind Tiwari, Jakov Vico and Craig Wester, "Motor Protection Principles, " IEEE 2008 